\title{
Sulfasalazine reduces placental secretion of antiangiogenic factors, up-regulates the secretion of placental growth factor and rescues endothelial dysfunction
}

\author{
Fiona C. Brownfoot ${ }^{\mathrm{a}, \mathrm{b}, *}$, Natalie J. Hannan ${ }^{\mathrm{a}, \mathrm{b}}$, Ping Cannon ${ }^{\mathrm{a}, \mathrm{b}}$, Vi Nguyen ${ }^{\mathrm{a}, \mathrm{b}}$, Roxanne Hastie ${ }^{\mathrm{a}, \mathrm{b}}$, Laura J. Parry ${ }^{\mathrm{c}}$, \\ Sevvandi Senadheera ${ }^{c}$, Laura Tuohey ${ }^{\mathrm{a}, \mathrm{b}}$, Stephen Tong ${ }^{\mathrm{a}, \mathrm{b}, 1}$, Tu'uhevaha J. Kaitu'u-Lino ${ }^{\mathrm{a}, \mathrm{b}, 1}$ \\ a Translational Obstetrics Group, Department of Obstetrics and Gynaecology, University of Melbourne, Mercy Hospital for Women, 163 Studley Road, Heidelberg 3084, Victoria, Australia \\ ${ }^{\mathrm{b}}$ Mercy Perinatal, Mercy Hospital for Women Heidelberg, Victoria, Australia \\ c School of BioSciences, University of Melbourne, Parkville 3010, Victoria, Australia
}

\section{A R T I C L E I N F O}

\section{Article history:}

Received 27 October 2018

Received in revised form 6 February 2019

Accepted 6 February 2019

Available online 26 February 2019

\section{Keywords:}

Preeclampsia

Endothelial dysfunction

Sulfasalazine

sFlt-1

\begin{abstract}
A B S T R A C T
Background: Preeclampsia is a major complication of pregnancy with no medical treatment. It is associated with placental oxidative stress, hypoxia and inflammation leading to soluble fms-like tyrosine kinase 1 (sFlt-1) and soluble endoglin (sENG) secretion and reduced placental growth factor (PIGF). This results in widespread endothelial dysfunction causing hypertension and multisystem organ injury. Sulfasalazine is an anti-inflammatory and antioxidant medication used to treat autoimmune disease. Importantly, it is safe in pregnancy. We examined the potential of sulfasalazine to quench antiangiogenic factors and endothelial dysfunction and increase angiogenic factor secretion.

Methods: We performed functional experiments using primary human pregnancy tissues to examine the effects of sulfasalazine on sFlt-1, sENG and PIGF secretion. Sulfasalazine is known to inhibit nuclear factor kappa-lightchain-enhancer of activated B cells (NFkB) and upregulate heme-oxygenase 1 (HO-1) thus we explored the effect of these transcription factors on sFlt-1 secretion from human cytotrophoblasts. We examined the ability of sulfasalazine to reduce key markers of endothelial dysfunction and dilate whole blood vessels.

Findings: We demonstrate sulfasalazine administration reduces sFlt- 1 and sENG and upregulates PIGF secretion from human placental tissues. Furthermore sulfasalazine mitigates endothelial dysfunction in several in vitro/ ex vivo assays. It enhanced endothelial cell migration and proliferation, promoted blood vessel dilation (vessels obtained from women at caesarean section) and angiogenic sprouting from whole blood vessel rings. The effect of sulfasalazine on the secretion of sFlt-1 was not mediated through either the NFkB or HO-1 pathways. Interpretation: We conclude that sulfasalazine reduces sFlt-1 and sENG secretion and endothelial dysfunction and upregulates PIGF. Sulfasalazine has potential to treat or prevent preeclampsia and warrants investigation in clinical trials.

Funding: This work was funded by The National Health and Medical Research Council of Australia (NHMRC; \#1048707, \#1046484. \#1101871, \#1064845), an Arthur Wilson RANZCOG scholarship and a Norman Beischer Medical Research Foundation grant. FB was supported by a NHMRC Early Career Fellowship (NHMRC \#1142636). NJH was supported by a CR Roper Research Fellowship. The NHMRC provided salary support (\#1136418 to ST \#1062418 to TKL, \#1064845 to SS). The funders had no role in study design, data collection, analysis, decision to publish or the preparation of the manuscript.
\end{abstract}

Crown Copyright $\odot 2019$ Published by Elsevier B.V. This is an open access article under the CC BY-NC-ND license (http://creativecommons.org/licenses/by-nc-nd/4.0/).

\footnotetext{
* Corresponding author at: Translational Obstetrics Group, University of Melbourne Mercy Hospital for Women, 163 Studley Rd., Heidelberg 3084, Victoria, Australia. E-mail address: Fiona.brownfoot@unimelb.edu.au (F.C. Brownfoot).

${ }^{1}$ Equal contribution
}

\section{Introduction}

Preeclampsia affects $5-8 \%$ of pregnancies and is annually responsible for 60,000 maternal deaths and over 500,000 stillbirths or neonatal deaths $[1,2]$. Key aspects in the pathophysiology of preeclampsia are the presence of placental oxidative stress, inflammation and hypoxia. This is accompanied by an excess secretion of the anti-angiogenic factors soluble fms-like tyrosine kinase 1 (sFlt-1) [3] and soluble endoglin 


\section{Research in context}

\section{Evidence before this study}

Preeclampsia is a serious complication of pregnancy with no medical treatment. There has been recent interest in repurposing medications that upregulate innate placental anti-oxidants and reduce hypoxic regulated transcription factors to treat preeclampsia. We have studied statins and identified esomeprazole and metformin as possible therapeutics for preeclampsia as they reduce placental sFlt-1 secretion and reverse features of endothelial cell dysfunction. Here in we identify sulfasalazine, a medication safe in pregnancy, as a possible therapeutic. Importantly its potent antiinflammatory properties accompanied by anti-oxidant effects identify it as an exciting and novel potential therapeutic. In this study we examined its effect on quenching key features of preeclampsia.

\section{Added value of this study}

This work identifies sulfasalazine, a medication safe in pregnancy, with anti-inflammatory and anti-oxidant properties as a possible treatment for preeclampsia. Using primary placental cells we demonstrate sulfasalazine reduces the secretion of sFIt-1 and sENG and increases PIGF. Furthermore using primary endothelial cells and omental vessels we show sulfasalazine mitigates vascular dysfunction and dilates whole vessels.

\section{Implications of all the available evidence}

Sulfasalazine is a potential treatment for preeclampsia and investigation in clinical trials is warranted.

(sENG) [4] into the maternal circulation and is accompanied by a reduction in the angiogenic factor placental growth factor (PIGF). This imbalance in antiangiogenic factors accompanied by increased inflammatory cytokines in the presence of widespread endothelial dysfunction leads to hypertension, multisystem organ injury or failure [5-7]. Currently, there is no medical treatment and delivery is the only way to stop disease progression [1]. A medical therapeutic that can stabilise the disease and reduce its severity would be a major advance, and could save many lives globally.

There has been recent interest in repurposing medications that upregulate innate placental anti-oxidant defences and reduce hypoxia. The medications that have been repurposed so far target placental hypoxia and upregulating anti-oxidant defences. An important part of the pathophysiology of preeclampsia is increased placental and systemic inflammation [14-17]. Increased circulating proinflammatory cytokines are likely to further exacerbate endothelial dysfunction. Therefore another approach in developing a treatment for preeclampsia would be to quench inflammation. In this study, we considered the potential of anti-inflammatory medication, sulfasalazine as a prevention or treatment for preeclampsia. Sulfasalazine has anti-inflammatory and immune modulating properties and potently induces anti-oxidants by upregulating the transcription factor HO-1 [18]. It is widely used to treat inflammatory arthritis and bowel disease and is considered safe to administer in pregnancy (FDA pregnancy category B). Sulfasalazine reduces cytokine secretion by inhibiting both transcription and the nuclear translocation of the inflammatory transcription factor NFKB [19]. It has been reported to reduce vascular neointimal hyperplasia likely through up-regulating endogenous anti-oxidant defences [18]. Therefore we reasoned that sulfasalazine might offer a prevention or treatment for preeclampsia with the added advantage of being an anti-inflammatory drug.
We examined whether sulfasalazine reduces sFlt-1 and sENG secretion, upregulate PIGF secretion and might rescue endothelial dysfunction in preeclampsia. If so, it may have the potential to treat or prevent preeclampsia. This study had three main objectives: 1) to assess the effects of sulfasalazine on sFlt-1, sENG and PIGF secretion from primary human placental and endothelial cells/tissues; 2) to explore whether the effects of sulfasalazine on sFlt- 1 and sENG secretion are mediated through its ability to induce $\mathrm{HO}-1$ or inhibit NFkB and 3 ) to assess whether sulfasalazine can reduce endothelial dysfunction, enhance dilation of human vessels and reverse sFlt-1 inhibition of angiogenesis in mouse aortic ring explants.

\section{Materials and methods}

Using primary human cells we performed functional experiments by administering sulfasalazine to examine its effect on key aspects of preeclampsia. Specifically we assessed the effect of sulfasalazine on placental secretion of sFlt-1, sENG and PIGF and on reducing markers of endothelial dysfunction on cells and in whole omental vessels. We also explored whether nuclear factor kappa-light-chain-enhancer of activated B cells (NFkB) and heme-oxygenase 1 (HO-1) affected sFlt-1 secretion using gene overexpression or silencing. To perform these experiments we firstly isolated primary cells.

\subsection{Isolating and treating primary human umbilical vein endothelial cells (HUVECS)}

We obtained umbilical cords from normal term placenta and the cord vein was infused with $10 \mathrm{ml}(1 \mathrm{mg} / \mathrm{ml})$ of collagenase (Worthington, Lakewood, New Jersey) and cells were isolated as previously described [20]. We used cells between passage 2 to 4 and we plated them at 24,000 cells $/ \mathrm{cm}^{2}$ and treated at $80 \%$ confluency with 0 , $200,500,1000 \mu \mathrm{M}$ sulfasalazine ( $\operatorname{cigma}$ ) $\mathrm{n}=3$ for $24 \mathrm{~h}$ at $37^{\circ} \mathrm{C}$ in $20 \%$ $\mathrm{O}_{2}$ and $5 \% \mathrm{CO}_{2}$. We collected conditioned media for assessment of sFlt1 , sENG and PIGF secretion and cell lysates for RNA and protein extraction.

\subsection{Isolating and treating primary human cytotrophoblast cells}

We collected term placentas from women having elective caesarean sections and isolated human cytotrophoblasts as previously described $[20,21]$. We plated cells at different cell densities and treated them for different periods of time as described below $(n=3)$.

We assessed the effect of sulfasalazine on cytotrophoblasts by plating cells at 24,000 cells $/ \mathrm{cm}^{2}$ and treating them with increasing doses of sulfasalazine at $0,50,100,200,300,500,750$ and $1000 \mu \mathrm{M}$ for $24 \mathrm{~h}$. We assessed the effect of gene silencing or gene overexpression by plating cells at 175,000 cells $/ \mathrm{cm}^{2}$ and 125,000 cells $/ \mathrm{cm}^{2}$ respectively. For silencing experiments, we transfected cytotrophoblasts with Lipofectamine RNAiMAX (life Technologies) and 1) 10 nM siRNA targeting NFkB (Qiagen) or $10 \mathrm{nM}$ siRNA targeting HO-1 (Qiagen) or 2) $16 \mu \mathrm{g} / \mathrm{ml}$ of IKBKB or NFKB p65 over expression plasmid and compared the output to a scrambled siRNA control (Qiagen) or an empty vector plasmid (Origene) respectively. We washed cells $24 \mathrm{~h}$ after treating them and changed the media every $48 \mathrm{~h}$ for a total treatment time of $96 \mathrm{~h}$. For the experiment assessing HO- 1 siRNA we treated the transfected cells with sulfasalazine at $750 \mu \mathrm{M}$ for the last $24 \mathrm{~h}$ of the experiment. All experiments were cultured at $37{ }^{\circ} \mathrm{C}$ in $8 \% \mathrm{O}_{2}$ and $5 \% \mathrm{CO}_{2}$.

We collected conditioned media from sulfasalazine, NFkB siRNA and overexpression plasmid (NFKB, p65 and IKBKB) experiments and assessed sFlt- 1 and sENG secretion and from the HO-1 siRNA experiment we assessed sFlt-1 secretion. We collected cell lysates for RNA and protein extraction. 


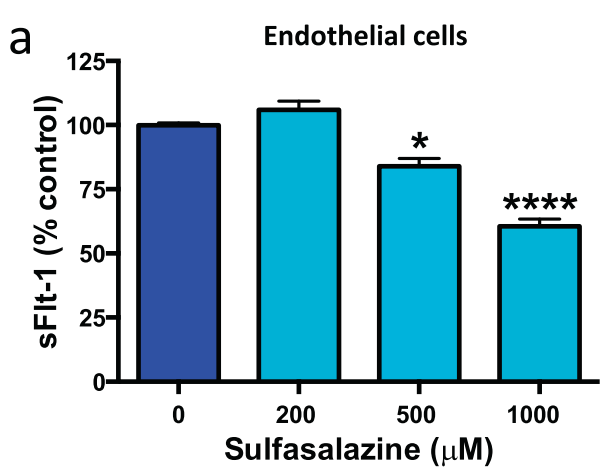

b

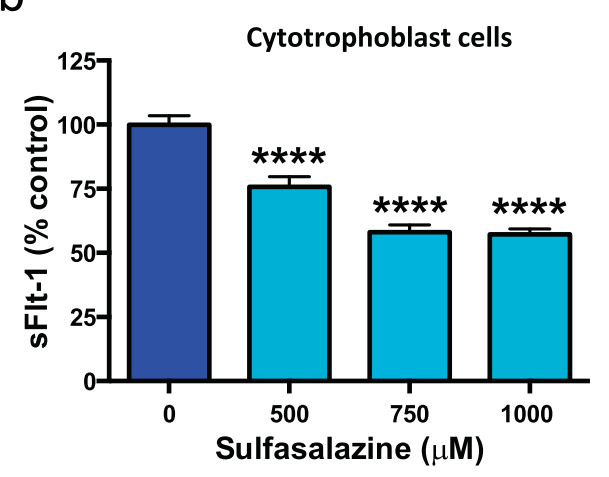

C

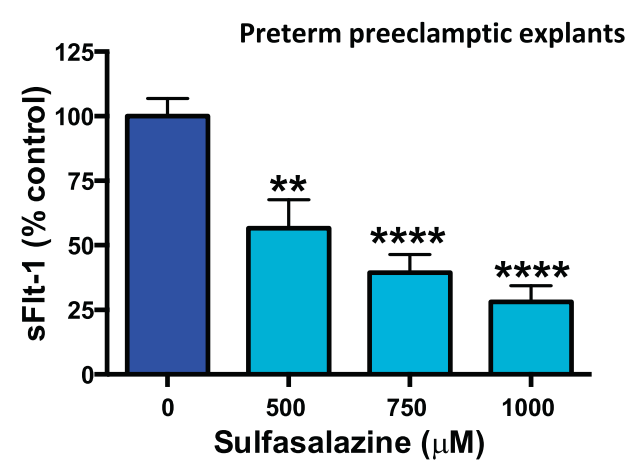

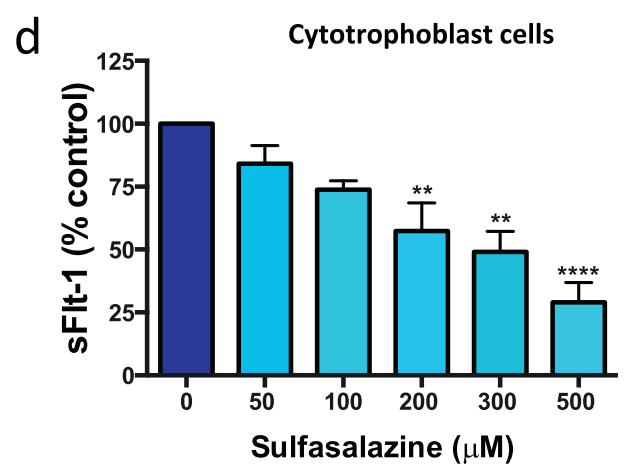

e

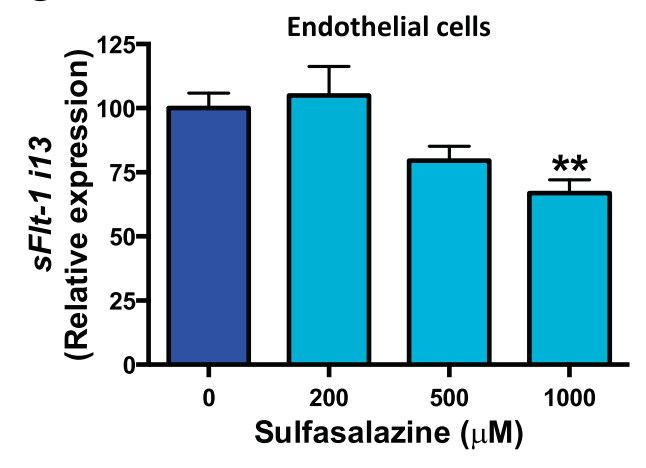

f

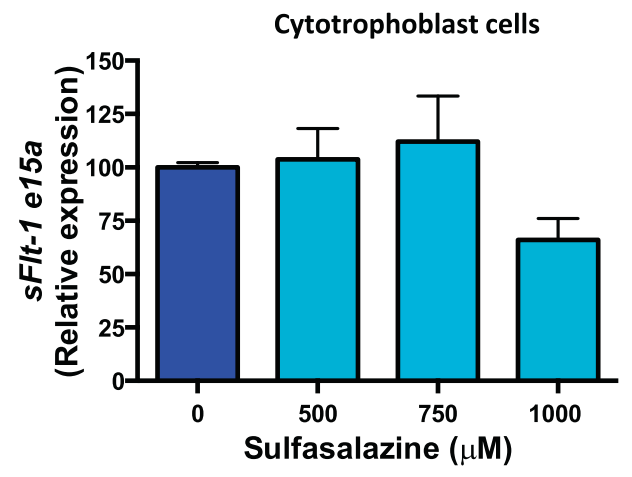

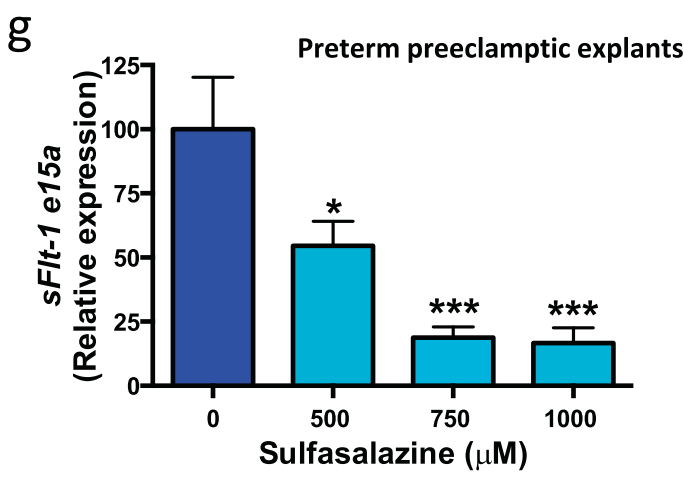

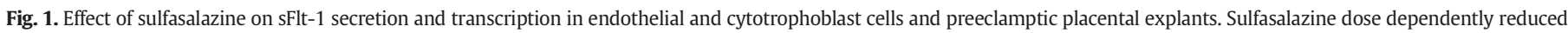

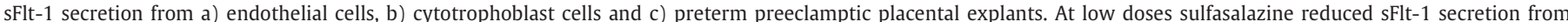

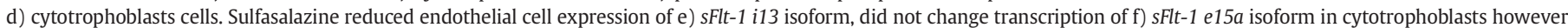

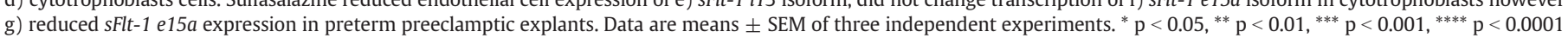
(Kruskal-Wallis). 
a

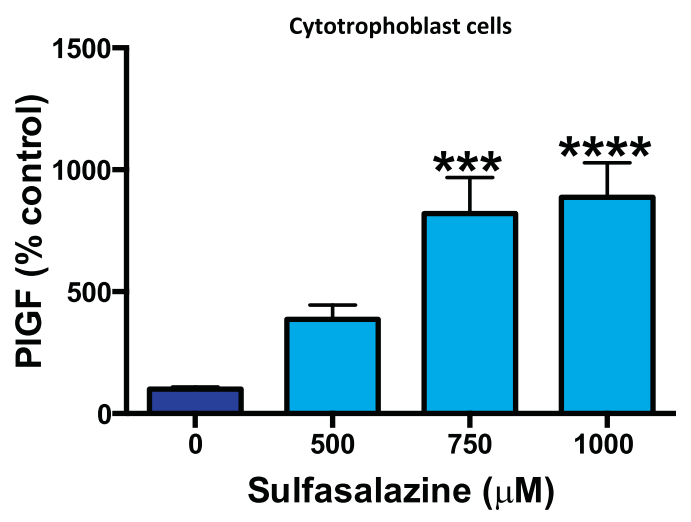

b

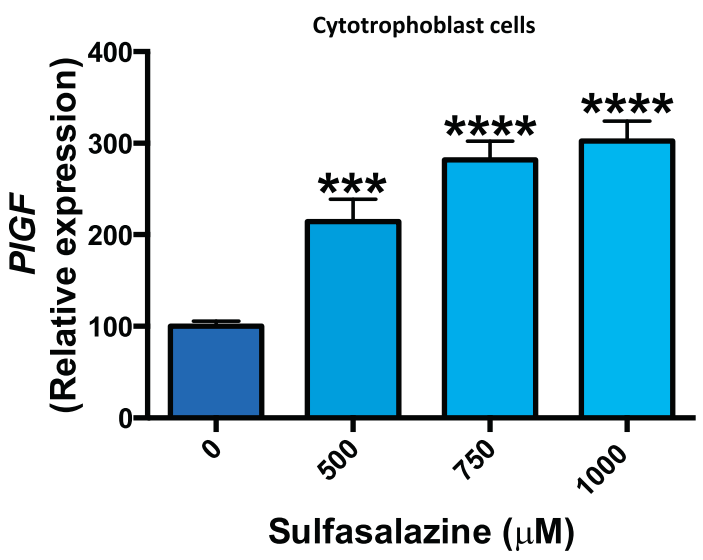

Fig. 2. Effect of sulfasalazine on PIGF secretion from cytotrophoblasts cells. Sulfasalazine dose dependently increased PIGF protein secretion a) and mRNA expression b) from cytotrophoblast cells. Data are means \pm SEM of three independent experiments. ${ }^{*} \mathrm{p}<$ $0.05,{ }^{* *} \mathrm{p}<0.01,{ }^{* * *} \mathrm{p}<0.001,{ }^{* * * *} \mathrm{p}<0.0001$ (Kruskal-Wallis).

\subsection{Culture of placental explants}

We collected human placental tissue from 4 women with early onset, proteinuric preeclampsia (delivered at $\leq 34$ weeks gestation). Preeclampsia was defined using the 2013 American College of Obstetricians and Gynecologists (ACOG) guidelines: the presence of hypertension $>140 / 90$ on two occasions $4 \mathrm{~h}$ apart and any of the following: proteinuria $>300 \mathrm{mg} /$ day, renal insufficiency, impaired liver function, thrombocytopenia or visual disturbance [22].

Preterm preeclamptic explants were prepared as previously described [20]. After we cultured the explants in media for $24 \mathrm{~h}$, we treated them with 500, 750, $1000 \mu \mathrm{M}$ sulfasalazine (sigma) for $24 \mathrm{~h}$ then re-treated and cultured them for a total of $72 \mathrm{~h}$ under $8 \% \mathrm{O}_{2}$ and $5 \% \mathrm{CO}_{2}$ at $37{ }^{\circ} \mathrm{C}$. We collected the conditioned media and assessed sFlt-1 and sENG levels. We normalized protein secretion against placental explant weights and collected the tissue for RNA extraction.

\subsection{Cell viability assay (MTS assay and calcein stain)}

We assessed cell viability using CellTiter 96-Aquesous One solution (Promega, Madison WI) assay according to the manufacturer's instructions or we stained the cells with calcein-AM (Merck Millipore, Darmstadt, Germany) and fluorescent signal was detected using Fluostar omega fluorescent plate reader (BMG labtech, Victoria, Australia).

\subsection{Endothelial dysfunction in primary HUVECS}

We induced endothelial dysfunction by treating primary HUVECs $(\mathrm{n}=4)$ with a constant dose of 1 ) $10 \mathrm{ng} / \mathrm{ml} \mathrm{TNF} \alpha$ (Sigma) or 2) by administering a pool of $10 \%$ serum obtained from three patients with preterm preeclampsia (comparison against $10 \%$ serum from a pooled sample from 3 gestationally matched normotensive controls). We administered increasing doses of sulfasalazine at 0, 200, 500, $750 \mu \mathrm{M}$ for $24 \mathrm{~h}$ at $20 \% \mathrm{O}_{2}, 5 \% \mathrm{CO} 2$ and $37{ }^{\circ} \mathrm{C}$. We collected lysates for RNA or protein extraction.

\subsection{Endothelial cell migration and proliferation}

We used the xCELLigence system (Roche diagnostic, New South Wales, Australia) to assess changes in endothelial cell migration and invasion. We used vascular endothelial growth factor (VEGF) (Bio-scientific, New South Wales, Australia) at $12.5 \mathrm{ng} / \mathrm{ml}$ as the chemoattractant in the lower well. We plated HUVECs between passage 2-4 at 160,000 cells $/ \mathrm{cm}^{2}$ in the upper well and treated them with $0 \mu \mathrm{M}$ or $200 \mu \mathrm{M}$ sulfasalazine to assess migration. We plated HUVECs between passage 2-4 were plated at 20,000 cells $/ \mathrm{cm}^{2}$ and treated with $0 \mu \mathrm{M}$ or $200 \mu \mathrm{M}$ sulfasalazine to assess proliferation. We measured electrical impedance for migration and proliferation in each well every minute for $2 \mathrm{~h}$ then every hour for $24 \mathrm{~h}$.

\subsection{Whole omental artery pressure myography}

We obtained omental arteries $(n=5)$ from patients undergoing elective caesarean section at term and prepared experiments as previously described [12]. Briefly, omental arteries were mounted on glass cannulae in a pressure myograph organ bath (Living Systems Instrumentation, Burlington, VT, USA) and pre-constricted with $0.1 \mu \mathrm{M} \mathrm{U} 46619$ (to reach $70 \%$ of maximum constriction). We treated omental arteries with sulfasalazine or vehicle (DMSO, dimethyl sulfoxide) at increasing increments $(0.1-3000 \mu \mathrm{M}$ with 2.5 min exposure at each dose) and the outer diameter of the vessel was assessed. The maximum final concentration of DMSO in the organ bath was $0.1 \%$.

\subsection{Mouse aortic ring explants}

We assessed the effects of sulfasalazine on angiogenesis ex vivo using mouse aortas that we scavenged and dissected as previously described [23]. Briefly, we cut the vessel into $0.5 \mathrm{~mm}$ rings and serum starved it in Optimem at $20 \% \mathrm{O}_{2}, 5 \% \mathrm{CO}_{2}$ at $37{ }^{\circ} \mathrm{C}$ overnight. We embedded it in a collagen matrix ( $1 \mathrm{mg} / \mathrm{ml}$ in DMEM, pH adjusted so slightly basic using $\mathrm{NaOH}$ ) in a 96 well plate with one mouse aortic explant per well. We treated the rings in media containing Optimem with $2.5 \%$ fetal calf serum (FCS) (Sigma, St Louis, United States) and antibiotic antimycotic $1 \%$ (Life Technologies) $\pm 250 \mathrm{ng} / \mathrm{ml} \mathrm{sFlt}-1$ and 0 or $25 \mu \mathrm{M}$ sulfasalazine (Sigma). We obtained $\mathrm{n}=5$ rings for each condition per mouse sample $n=4$. We changed the treatment every $48 \mathrm{~h}$ and continued the experiment for a total of $144 \mathrm{~h}$. We added calcein-AM (Merk Millipore) to the wells for $45 \mathrm{~min}$ at $37^{\circ} \mathrm{C}$ and images were obtained at the same magnification $(\times 40)$ using the EVOS FL microscope (Life Technologies). We determined vessel outgrowth by calculating area of growth using the Image J software (http://imagej.nih.gov/ij/) using a blinded observer.

\subsection{ELISA analysis}

We measured concentrations of sFlt-1, sENG and PIGF in conditioned cell/tissue culture media using the DuoSet VEGF R1/Flt-1 kit (R\&D systems by Bioscience, Waterloo, Australia), a DuoSet Human Endoglin CD/105 ELISA kit (R\&D systems) or Duoset Human PIGF kit (R\&D systems) according to manufacturer's instructions. 
a

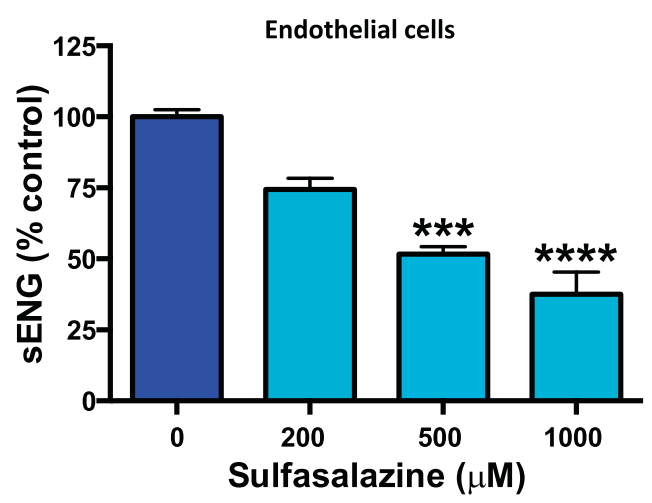

b

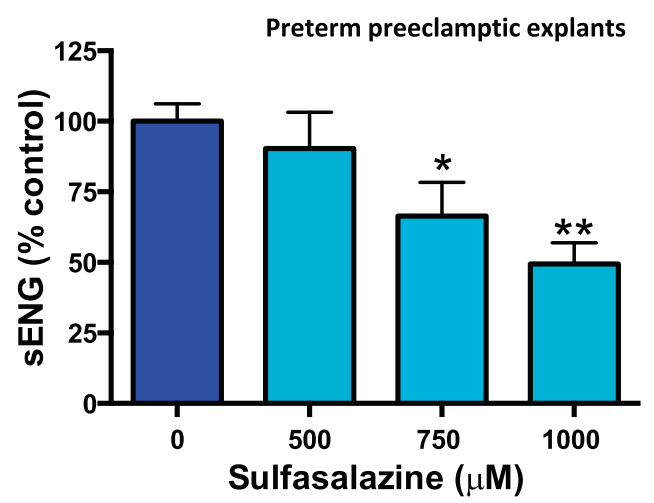

C

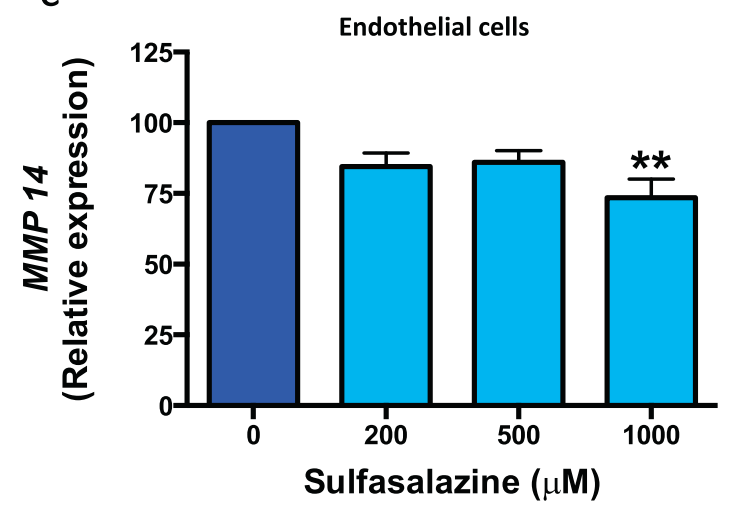

d

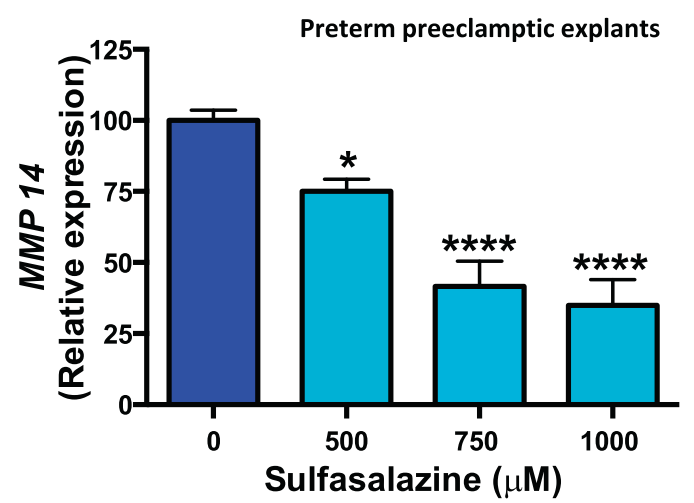

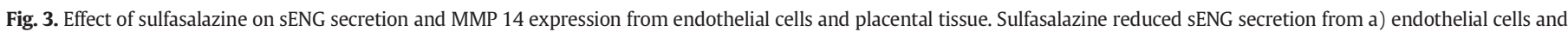

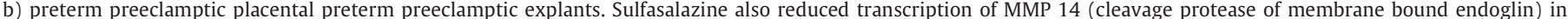

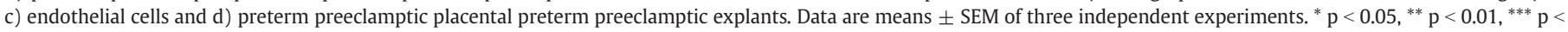
$0.001,{ }^{* * * *} \mathrm{p}<0.0001$ (Kruskal-Wallis).

\subsection{RT-PCR}

We extracted RNA from placental explants and HUVECs using an RNeasy mini kit (Qiagen, Valencia, CA) and quantified this using the Nanodrop ND 1000 spectrophotometer (NanoDrop technologies Inc., Wilmington, DE). We converted $0.2 \mu \mathrm{g}$ of RNA to cDNA using the Applied Biosystems high capacity cDNA reverse transcriptase kit (Life Technologies) as per manufacturer guidelines.

We assessed gene expressions of VCAM-1 (Life Technologies), MMP 14 (Life Technologies), PlGF (Life Technologies), HO-1 (Life Technologies), NFKB p65 (Life Technologies), IKBKB (Life Technologies) and $N F \kappa B$ (Life Technologies), Rel A (Life Technologies) and IKBKB (Life Technologies) by real time PCR (RT-PCR) on the CFX 384 (Bio-Rad, Hercules, CA) using FAM-labeled Taqman universal PCR mastermix and its specific primer/probe set (Life Technologies) with the following run conditions: $50{ }^{\circ} \mathrm{C}$ for $2 \mathrm{~min}$; $95{ }^{\circ} \mathrm{C}$ for $10 \mathrm{~min}, 95{ }^{\circ} \mathrm{C}$ for $15 \mathrm{~s}$, $60{ }^{\circ} \mathrm{C}$ for $1 \mathrm{~min}$ ( 40 cycles). SYBR RT-PCR was carried out to assess gene expressions of sFlt-1 e15a and sFlt-1 113 and GAPDH. We designed primers as previously described (Geneworks, South Australia, Australia) [24]. We performed quantitative RT-PCR using the run conditions: $95{ }^{\circ} \mathrm{C}$ for $20 \mathrm{~min}$; $95{ }^{\circ} \mathrm{C}$ for $0.01 \mathrm{~min}, 60{ }^{\circ} \mathrm{C}$ for $20 \mathrm{~min}, 95{ }^{\circ} \mathrm{C}$ for $1 \mathrm{~min}$ ( 39 cycles), melt curve $65{ }^{\circ} \mathrm{C}$ to $95{ }^{\circ} \mathrm{C}$ at $0.05{ }^{\circ} \mathrm{C}$ increments at $0.05 \mathrm{~s}$.
We normalized data to GAPDH, Topo or Cyt-c as a housekeeper and calibrated against the average $C_{t}$ of the control samples. Results were expressed as fold change from control.

\subsection{Western blot analysis}

We separated HUVEC or cytotrophoblast protein lysates (20 $\mu \mathrm{g})$ on $10 \%$ polyacrylamide gels with wet transfer to PVDF membranes (Millipore, Billerica, MA). We blocked membranes prior to incubation overnight with the primary antibody (Either anti-HO-1 at 1:500 dilution (ADI-SPA-896 (ENZO Life Sciences, Farmingdate, New York) or anti-VCAM 1 at 1:200 dilution (SC-1504), Santa Cruz, Biotechnology, Dallas, Texas)). We assessed heme-oxygenase 1 (HO-1) by blocking blots with $5 \%$ skim milk followed by incubation with primary rabbit anti-HO-1 antibody (1:500) overnight at $4{ }^{\circ} \mathrm{C}$, and incubated them with secondary anti-rabbit-HRP antibody for $1 \mathrm{~h}$ at room temperature. We examined vascular cell adhesion molecule 1 (VCAM 1) by blocking protein blots with $5 \%$ skim milk, followed by incubation with primary goat anti-VCAM 1 antibody $(1: 200)$ in Canget 1 overnight at $4{ }^{\circ} \mathrm{C}$ then incubated with secondary anti goat-HRP antibody for $1 \mathrm{~h}$ at room temperature. We applied ECL prime reagent (GE healthcare Life Sciences, Pittsburgh, PA) and immune-reactive bands were visualized using a Chemidoc XRS (BioRad, Hercules, CA). We stripped blots with Restore 


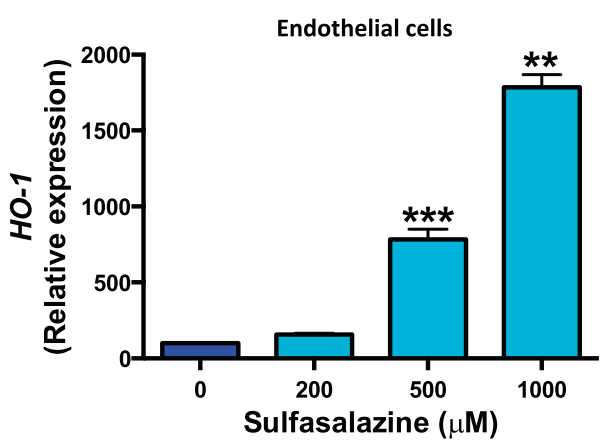

b Endothelial cells
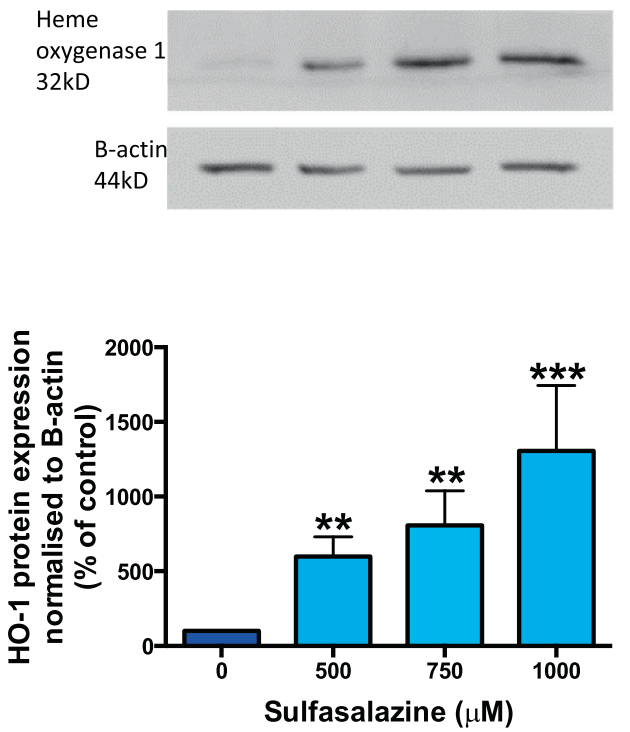

e

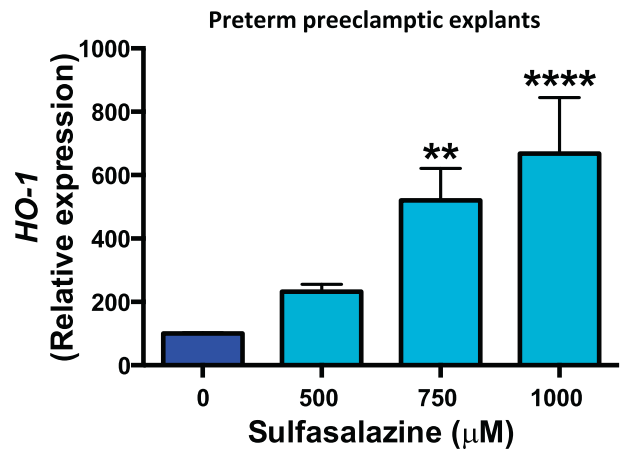

C

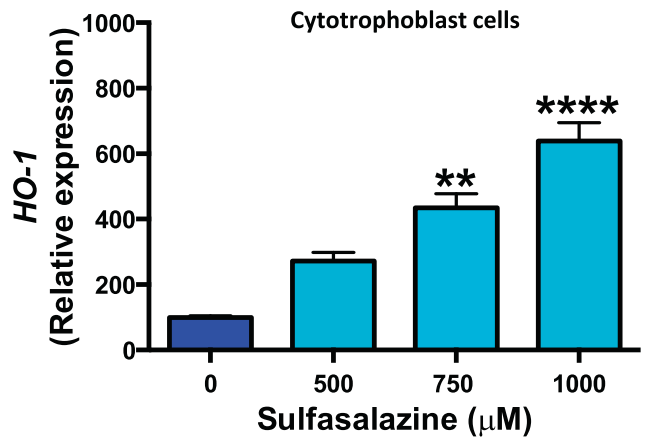

d Cytotrophoblast cells
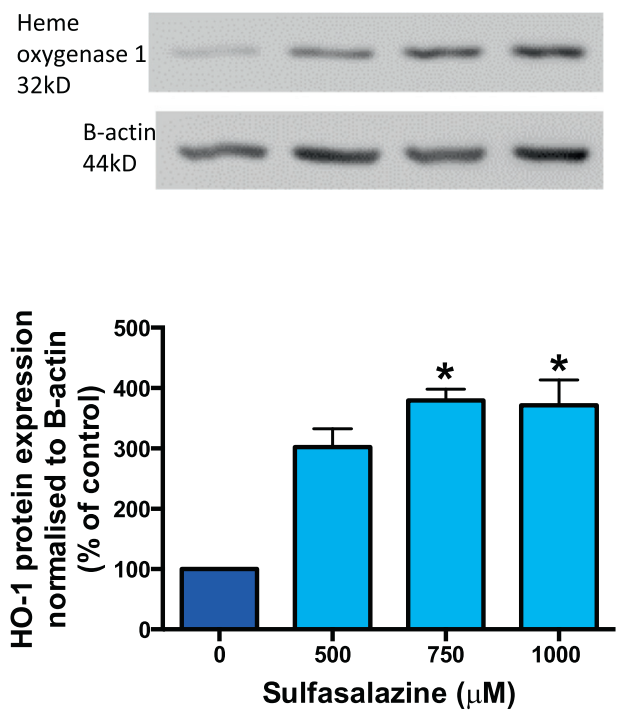

f

Cytotrophoblast cells

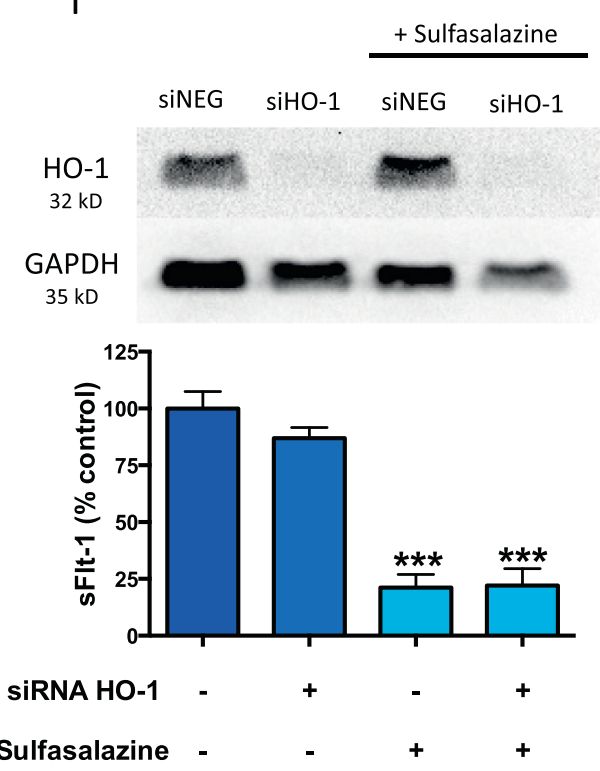

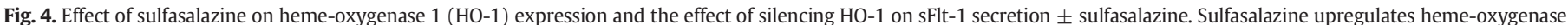

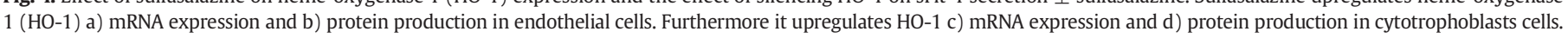

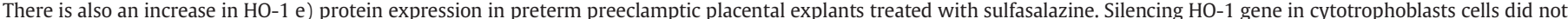

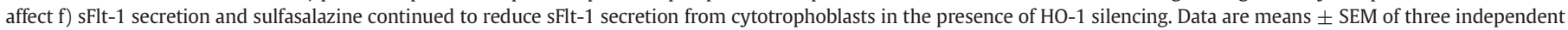
experiments. $\mathrm{p}<0.05,{ }^{* *} \mathrm{p}<0.01,{ }^{* * *} \mathrm{p}<0.001,{ }^{* * * *} \mathrm{p}<0.0001$. (Kruskal-Wallis). 
a

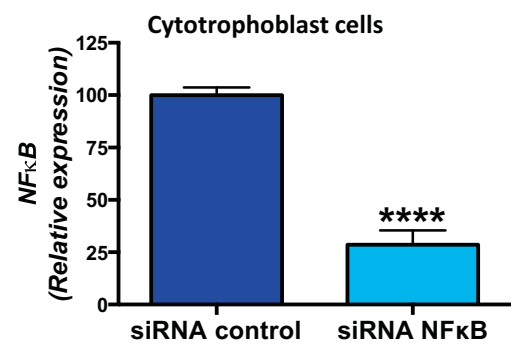

C
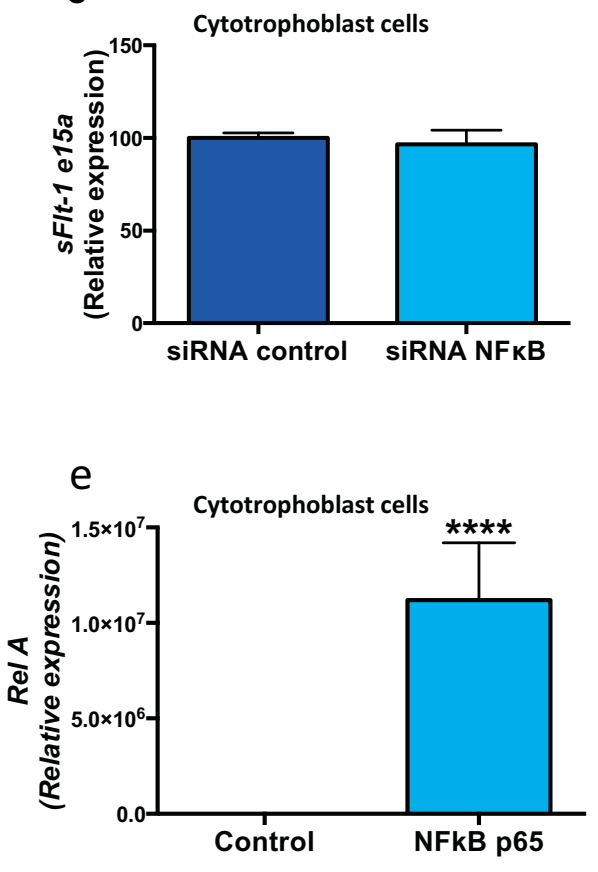

g

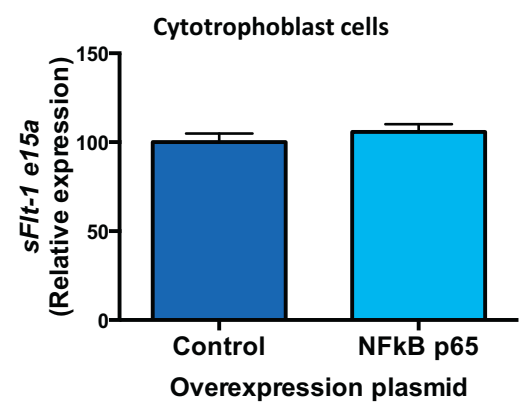

b

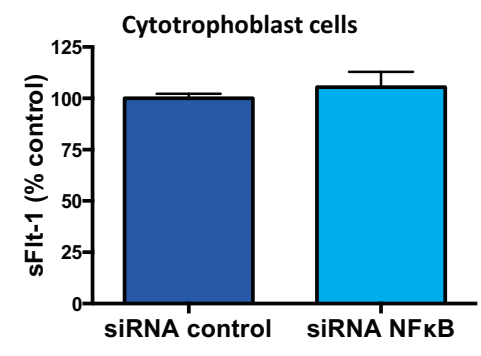

d

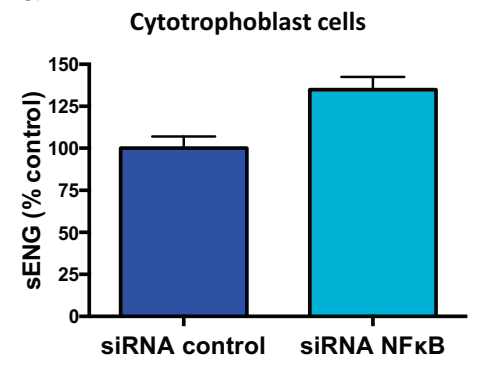

$f$

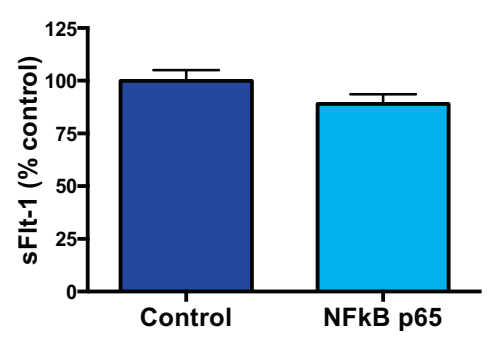

$\mathrm{h}$

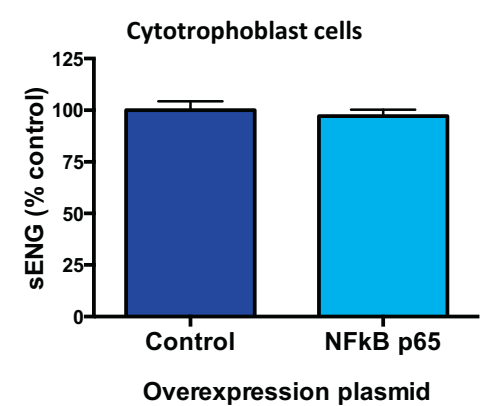

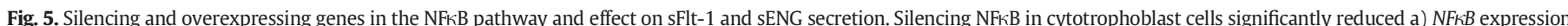

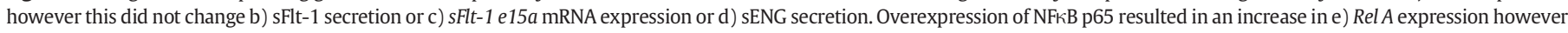

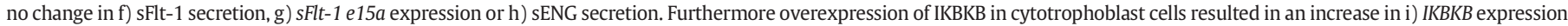

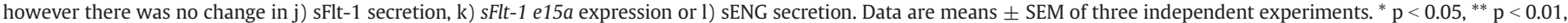
*** $\mathrm{p}<0.001,{ }^{* * * *} \mathrm{p}<0.0001$ ( $t$-test). 

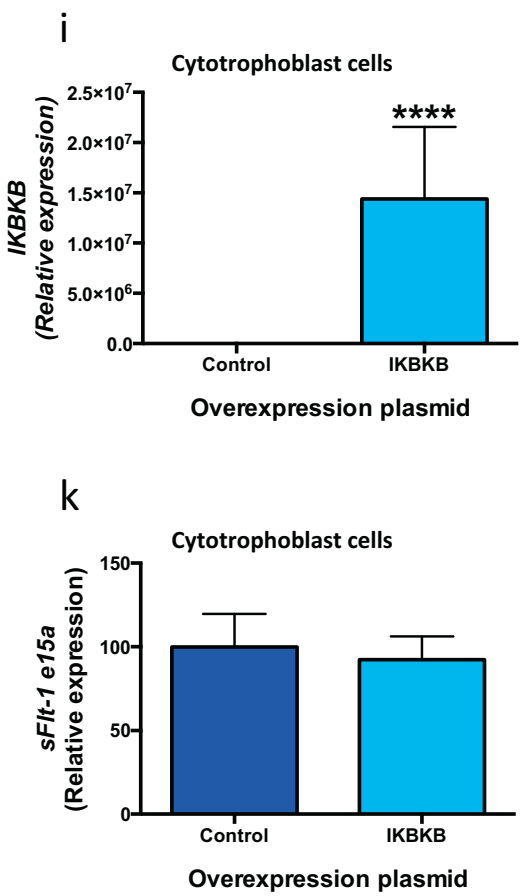

j

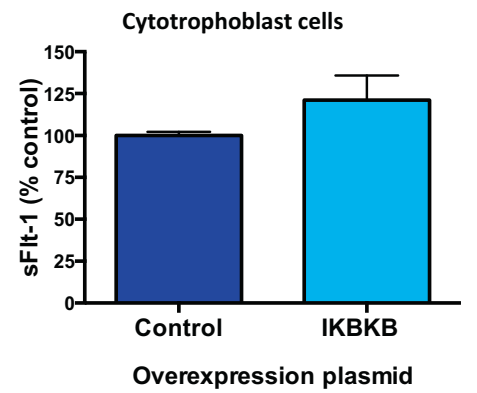

I

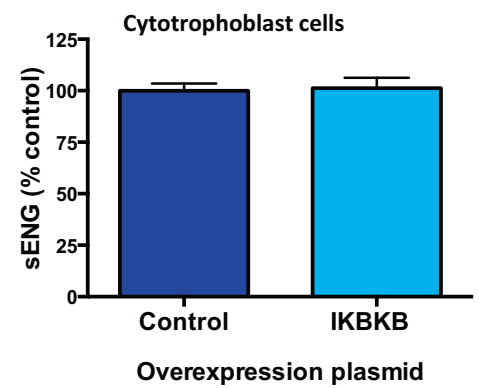

Fig. 5 (continued)

Western Blot Stripping Buffer (Thermo Fisher Scientific, Waltham, MA) and re-probed it with mouse anti-B-actin $(1: 10,000)$ antibody overnight at $4{ }^{\circ} \mathrm{C}$ for the protein loading control.

\subsection{Ethics approval}

Our study was approved by The Mercy Health Human Research Ethics Committee (Institutional review board number R11/34 and R14/11) and all women gave written informed consent. We sourced mouse aortas from animals after sacrifice in ethically approved experiments conducted by other research groups within the Institute.

\subsection{Statistical analysis}

We performed a minimum of three technical triplicates for each biological replicate, with a minimum of three biological replicates (each from different patients) performed for each in vitro study. We assessed two groups using a $t$-test (parametric) or a Mann-Whitney test (nonparametric) and a 1-way ANOVA (parametric) or a Kruskal-Wallis test (non-parametric) when three or more groups were compared. We used the GraphPad Prism 6 (GraphPad Software, La Jolla, CA) for statistical analysis. ' $n$ ' represents the number of different patients used during the whole omental vessel experiments. We fitted the concentration response curves from omental arteries to a sigmoidal curve with nonlinear regression to calculate the sensitivity for sulfasalazine $\left(\mathrm{pEC}_{50}\right)$ or maximum relaxation $\left(E_{\max }\right)$ as previously described [12]. We analysed the response curves using two-way repeated measures ANOVA with Bonferroni posthoc analysis (treatment versus concentration). All data were expressed as mean \pm SEM; P values $<0.05$ were considered significant.

\section{Results}

3.1. Effect of sulfasalazine on sFlt-1 secretion from primary HUVECs and placental tissues

To examine the effect of sulfasalazine on sFlt-1 secretion we obtained primary human placental tissues. We isolated human umbilical vein endothelial cells (HUVECs) and cytotrophoblasts from the placenta (as endothelial and trophoblast cells are the main source of sFlt-1) using a protocol previously described [25]. Placental explants were also examined from patients with preterm preeclampsia. Sulfasalazine dose dependently reduced sFlt-1 secretion from primary HUVECs (Fig. 1a). Sulfasalazine also dose dependently reduced sFlt-1 secretion from cytotrophoblasts (Fig. 1b) and placental explants obtained from women with preterm preeclampsia (Fig. 1c). We explored the effect of low doses of sulfasalazine on cytotrophoblasts and there was a dose dependant reduction in sFlt-1 secretion (Fig. 1d).

We investigated the effect of sulfasalazine on the transcription of sFlt-1 splice variants (e15a and i13) [26,27] in HUVECs, cytotrophoblast and preterm preeclamptic placental explants. sFlt-1 i13 is the most abundantly expressed isoform in endothelial cells [26]. Addition of sulfasalazine to HUVECs significantly reduced sFlt-1 13 transcription (Fig. 1e). sFlt-1 e15a is the dominant sFlt-1 isoform expressed in placenta [26]. Whilst sulfasalazine did not change sFlt-1 e15a transcription in cytotrophoblasts (Fig. 1f), there was a dose dependent reduction in transcription in preeclamptic placental explants (Fig. 1g). Therefore, we conclude sulfasalazine reduces sFlt-1 secretion from HUVECs and placental cells/tissues, including placental explants obtained from patients with preterm preeclampsia.

\subsection{Effect of sulfasalazine on placental growth factor (PIGF) secretion from primary human tissues}

Placental growth factor is a circulating pro-angiogenic protein secreted from the placenta that is neutralised by sFlt- 1 and reduced in preeclampsia. We explored the effect of sulfasalazine on PIGF secretion and expression from primary cytotrophoblasts. With increasing doses of sulfasalazine there was a dose dependant increase in PIGF protein secretion (Fig. 2a) and this corresponded to a significant increase in PlGF mRNA expression (Fig. 2b).

\subsection{Effect of sulfasalazine on sENG secretion in primary endothelial cells and placental tissues}

Soluble endoglin is an anti-angiogenic factor produced from cleavage of membrane bound endoglin and is increased in preeclampsia. 
a

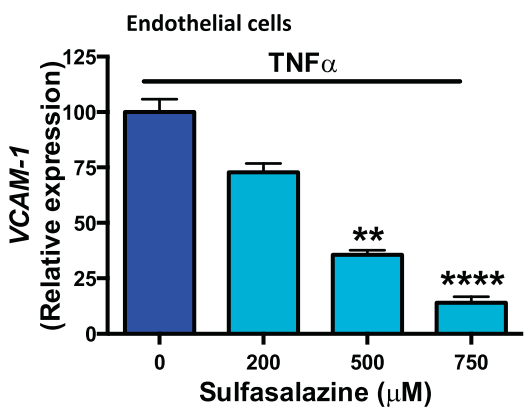

C

Endothelial cells

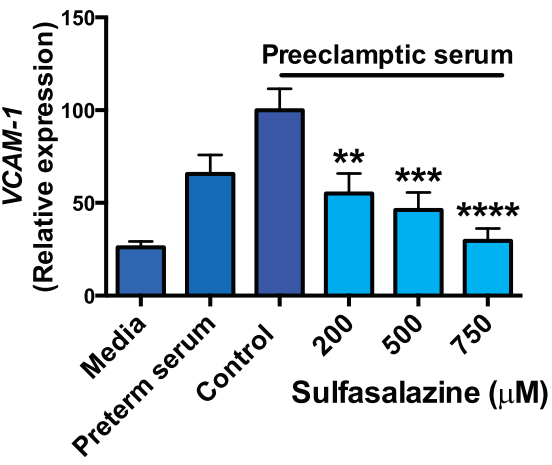

Endothelial cells

b
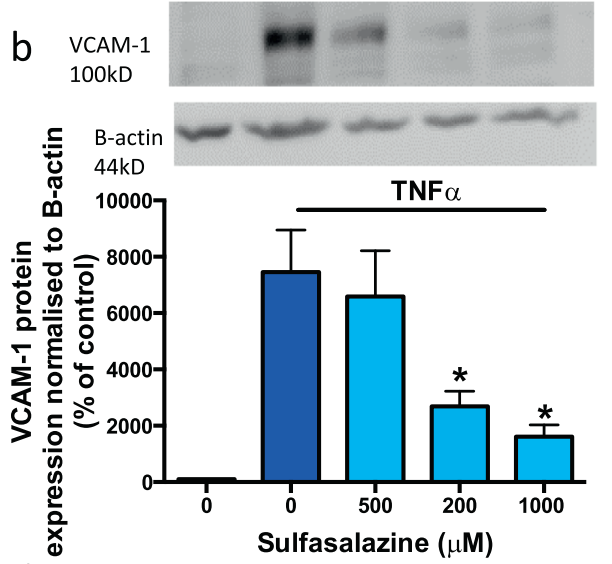

d

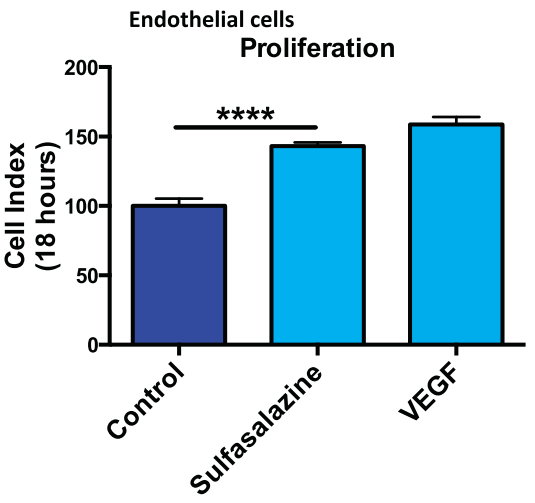

e

Endothelial cells
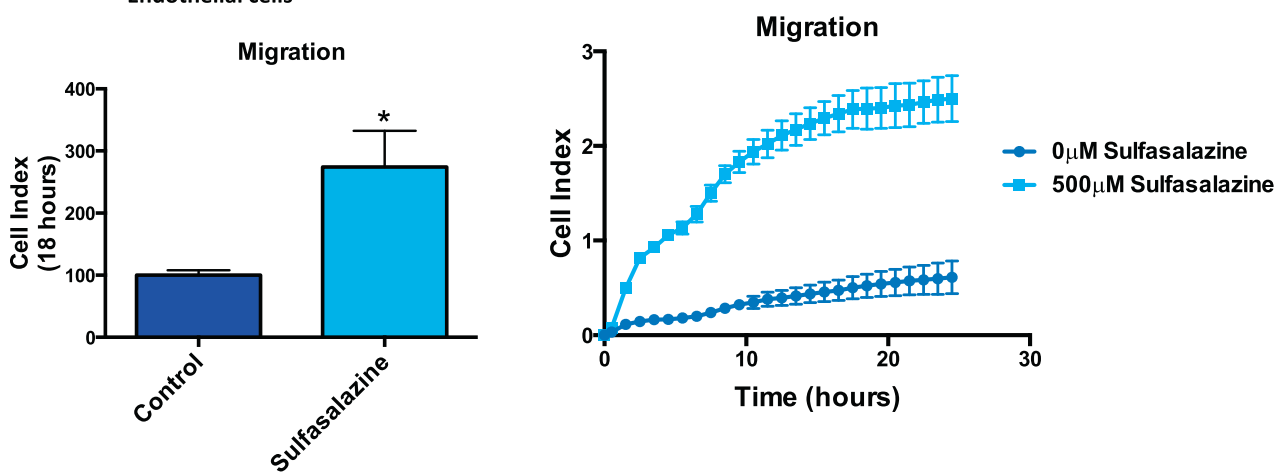

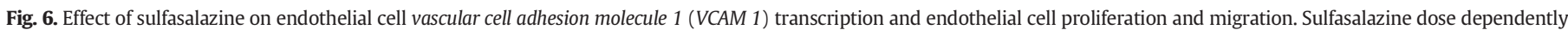

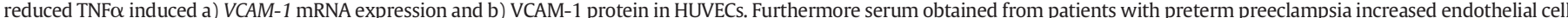

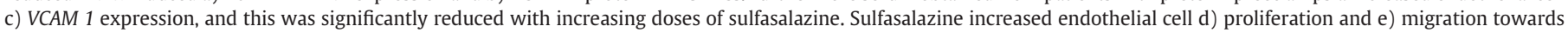
chemoattractant vascular endothelial growth factor. Data are means \pm SEM of three independent experiments. ${ }^{*} \mathrm{p}<0.05,{ }^{* *} \mathrm{p}<0.01,{ }^{* * *} \mathrm{p}<0.001,{ }^{* * * *} \mathrm{p}<0.0001$. (Kruskal-Wallis).

Next we investigated the effects of sulfasalazine on sENG secretion. Sulfasalazine dose dependently reduced sENG secretion from HUVECs (Fig. 3a) and from placental explants obtained from patients with preterm preeclampsia (Fig. 3b).

MMP 14 is a membrane bound protease, responsible for the release of sENG into the circulation [28]. We examined the effect of sulfasalazine on the expression of MMP 14 mRNA in the same cells/tissues where sENG was reduced. Sulfasalazine reduced MMP 14 mRNA expression in both HUVECs (Fig. 3c) and preterm preeclamptic placental explants (Fig. 3d).

\subsection{Effect of sulfasalazine on heme-oxygenase 1 in primary human tissues}

Given sulfasalazine has been shown to induce HO-1 (a molecule with anti-oxidant properties) in vascular smooth muscle cells [18] we explored whether sulfasalazine might also up-regulate $\mathrm{HO}-1$ in placenta. Indeed, we found sulfasalazine dose dependently increased $\mathrm{HO}-1$ expression (Fig. 4a) and protein (Fig. 4b) in HUVECs. Additionally sulfasalazine increased $\mathrm{HO}-1$ mRNA (Fig. 4c) and protein (Fig. 4d) in cytotrophoblast, and in preterm preeclamptic placental explants (Fig. 4e). 

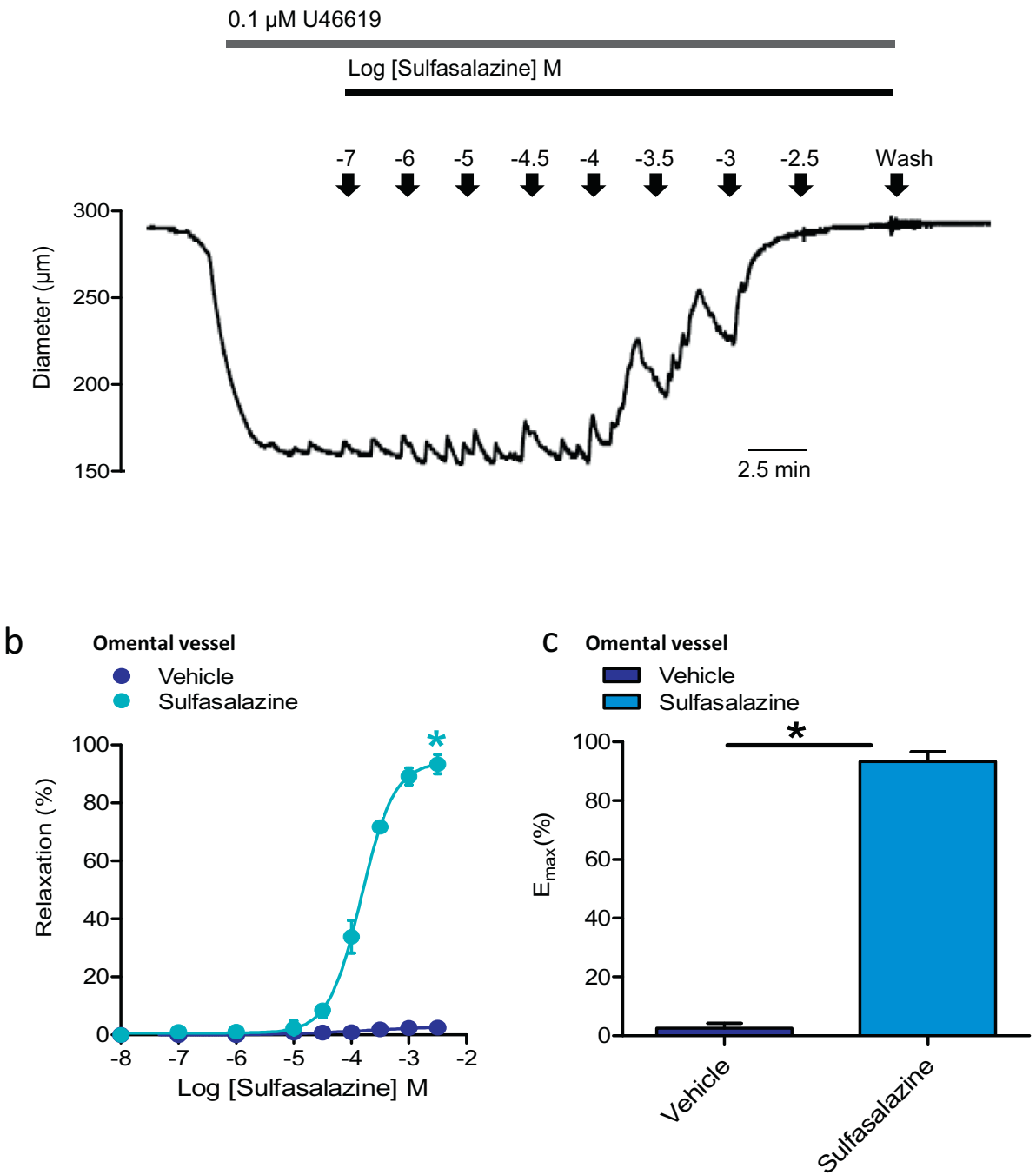

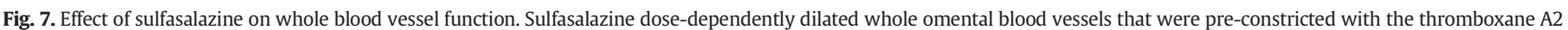

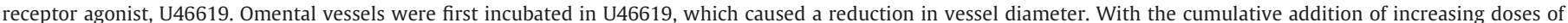

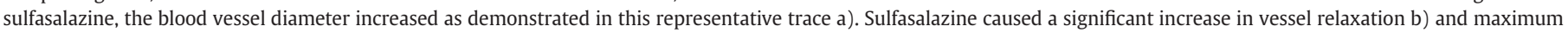
relaxation $($ Emax $)$ c) compared with vehicle (DMSO) controls. Data are mean \pm SEM of three independent experiments. ${ }^{*} \mathrm{p}<0.05$ ( $\mathrm{t}$-test).

We explored whether the effects of sulfasalazine on sFlt-1 secretion were mediated by its ability to induce HO-1 expression. To do this, we concurrently silenced HO-1 expression in cytotrophoblasts and added sulfasalazine. Despite efficient HO-1 silencing, the addition of sulfasalazine consistently reduced sFlt-1 secretion (Fig. 4f). Notably, efficient silencing of HO-1 in the absence of sulfasalazine did not affect sFlt-1 secretion (Fig. 4f), which is consistent with the findings of our previous report which showed, HO-1 does not directly regulate sFlt-1 production [29]. Thus, it appears that the reduction in sFlt-1 secretion from cytotrophoblasts induced by sulfasalazine is not mediated through HO-1.

3.5. Effect of inhibiting and overexpressing molecules in the NFkB pathway on sFlt-1 and sENG secretion

Sulfasalazine is known to inhibit the inflammatory transcription factor NFkB [6], likely through stabilising proteins that stop its translocation into the nucleus [19]. Given that sulfasalazine has such effects on $\mathrm{NF} \kappa \mathrm{B}$ activity, and that it also reduces sFlt- 1 and sENG secretion, we examined whether the NFkB pathway regulates sFlt- 1 and sENG secretion. We silenced NFKB in cytotrophoblasts using siRNA ( reduced its mRNA expression by $71 \%$ (Fig. $5 \mathrm{a}$ ). siNFkB did not alter the secretion of sFlt-1 (Fig. 5b), expression of sFlt-1 e15a (Fig. 5c) or sENG (Fig. 5d). Similarly, overexpressing NFkB p65 in cytotrophoblasts upregulated the p65 subunit Rel A (Fig. 5E), did not change sFlt-1 secretion (Fig. 5f), sFlt-1 e15a expression (Fig. 5g) or sENG secretion (Fig. 5h). Furthermore, overexpressing IKBKB increased IKBKB (Fig. 5i), however did not alter sFlt-1 secretion (Fig. 5j), sFlt-1 e15a expression (Fig. 5k) or sENG secretion (Fig. 5l). Taken together, our data suggests the NFkB pathway does not regulate sFlt-1 or sENG secretion from placental cytotrophoblasts.

3.6. Effect of sulfasalazine on VCAM-1 transcription and protein and the proliferation and migration of HUVECS

Endothelial dysfunction is associated with increased expression of vascular cell adhesion molecule-1 (VCAM-1) [30,31]. VCAM-1 is upregulated by pro-inflammatory stimuli and is expressed on the luminal surface of blood vessels where it can bind and sequester leukocytes, erythrocytes and platelets. VCAM- 1 expression is induced by proinflammatory stimuli. Preeclampsia is associated with increased circulating TNF $\alpha$ [15], a pro-inflammatory cytokine that up-regulates 
a Mouse aortic rings

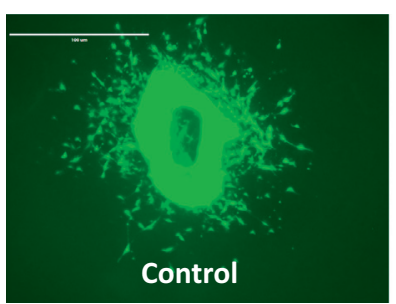

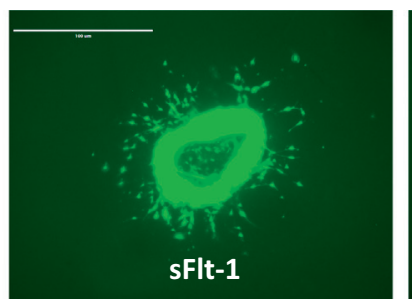

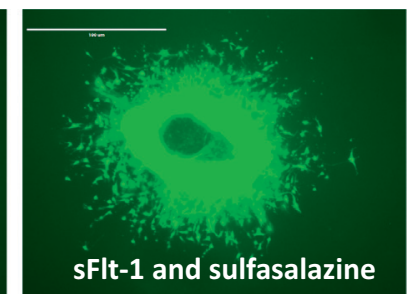

b Mouse aortic rings

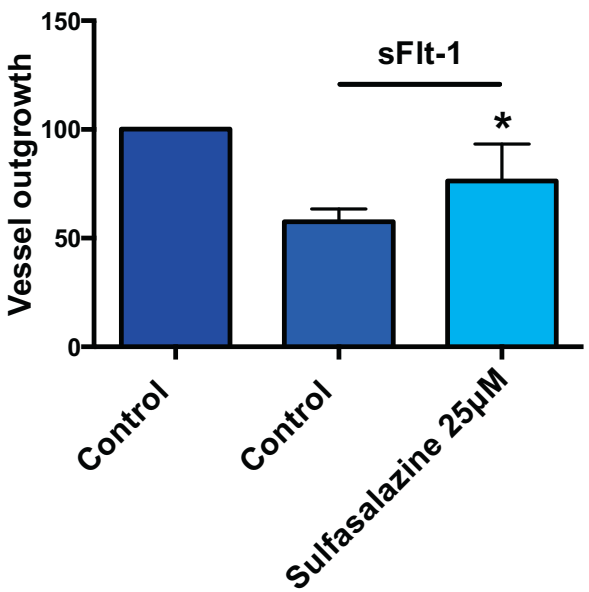

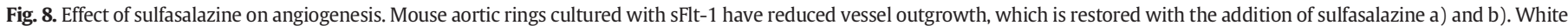
arrows point at vessel outgrowth. Representative micrographs are shown. Data are means $\pm \mathrm{SEM}$ of three independent experiments. ${ }^{*} \mathrm{p}<0.05$ ( $\mathrm{t}$-test).

VCAM-1 [30,31]. We therefore performed an in vitro endothelial dysfunction assay where HUVECs were treated with TNF $\alpha$ to upregulate VCAM-1 and then co-administered sulfasalazine. Sulfasalazine potently reduced the TNF $\alpha$ induced VCAM-1 mRNA expression (Fig. 6a) and VCAM-1 protein (Fig. 6b). Next, we administered pooled serum from three patients with preterm preeclampsia to primary HUVECs, which induced a significant increase in VCAM-1 expression (Fig. 6C) that was quenched with the addition of sulfasalazine (Fig. 6c).

We then examined whether sulfasalazine affected HUVEC proliferation and migration by monitoring cells using the xCELLigence system (measures experiments in real time). We demonstrated sulfasalazine increased HUVEC proliferation (Fig. 6d) and enhanced HUVEC migration towards the chemoattractant VEGF (Fig. 6e and f).

Thus, sulfasalazine reduced VCAM-1 in HUVECs in two in vitro endothelial dysfunction models and enhanced endothelial proliferation and migration. These data suggest sulfasalazine rescues endothelial dysfunction.

\subsection{Effect of sulfasalazine on whole maternal vessel dilation isolated from the omentum}

In order to determine if sulfasalazine affected vessel diameter, we undertook whole artery functional experiments. Arteries were isolated from omental tissue obtained from women having a caesarean section. Arteries were then subjected to pressure myography to examine vascular function. As expected, the administration of the thromboxane A2 receptor agonist U46619 caused constriction of the vessel (reduced arteriole diameter). However, in the presence of sulfasalazine, this was significantly augmented with an increase in relaxation $\left(\mathrm{pEC}_{50} 150 \pm 8\right.$ $\mu \mathrm{M}, \mathrm{E}_{\max } 93.4 \pm 3.3 \%$ ) compared to the vehicle controls, an effect that was dose dependent (Fig. 7 a, b and c). This suggests that sulfasalazine can induce dilation in a pre-constricted artery.

\subsection{Sulfasalazine effect on angiogenic sprouting from mouse aorta}

Preeclampsia is believed to be associated with decreased angiogenesis [5,32]. We explored whether sulfasalazine could rescue sFlt- 1 induced inhibition of angiogenesis by performing a mouse aortic angiogenesis assay [23]. Mouse aortic explants were cultured in the presence of recombinant human sFlt- $1 \pm$ sulfasalazine (Fig. 8 a and b). Administering sFlt-1 alone reduced de novo angiogenic sprouting from the aortic rings. Sulfasalazine rescued the inhibition of angiogenic sprouting seen with the addition of sFlt-1, suggesting that it may have pro-angiogenic actions (Fig. 8 a and b).

\section{Discussion}

Preeclampsia is one of the most serious complications of pregnancy and can place the life of mother and fetus at risk. Currently, there is no medical treatment and delivery is the only way to stop disease progression. At early gestations, the need to deliver to save the mother can inflict complications of prematurity on the infant including death, cerebral palsy, chronic lung disease and intracerebral bleeding [33]. The risks for mothers and babies continue throughout life as they are at increased risk of cardiovascular morbidity and mortality from ischaemic heart disease and stroke [34-36].

Our group and others have demonstrated statins reduce sFlt- 1 secretion from cell lines [8] and from primary human placental tissues and pravastatin also reverses key features of endothelial dysfunction $[9,10]$. We identified esomeprazole [11] and metformin [12] as 
medications that reduce sFlt- 1 and sENG secretion from primary placental cells and tissues and mitigate key aspects of endothelial dysfunction in primary endothelial cells and whole vessels. Given these promising results these medications have progressed to randomised clinical trial. The StAmP trial (A Proof of Principle, Double-Blind, Randomised, Placebo-Controlled, Multi Centre Trial of pravaStatin to Ameliorate Early Onset Pre-eclampsia) has finished recruitment in the United Kingdom and we await the results of its primary outcome assessing the levels of sFlt- $148 \mathrm{~h}$ post randomisation. In collaboration with colleagues in South Africa we have completed the PIE (Preeclampsia Intervention with Esomeprazole) trial randomising 120 women with preterm preeclampsia to esomeprazole or placebo. There was no difference in our primary outcome of gestation prolongation [13]. Following on from this trial we have recently commenced the PI2 (Preeclampsia Intervention 2) trial examining metformin as a treatment for preeclampsia and we will recruit 150 women with preterm preeclampsia to see whether metformin might prolong gestation.

We present a body of work suggesting sulfasalazine may be a promising prevention or treatment for preeclampsia. We have performed multiple functional assays using primary human tissues to demonstrate sulfasalazine may mitigate key features involved in the pathophysiology of preeclampsia. Firstly, we demonstrate sulfasalazine reduced sFlt-1 and sENG secretion from primary endothelial cells, cytotrophoblast cells and placental explants obtained from cases of preterm preeclampsia. This reduction in sFlt- 1 secretion does not appear to be mediated through the HO- 1 or NFkB pathways. We also demonstrate sulfasalazine can increase PlGF expression and secretion from cytotrophoblasts. Furthermore sulfasalazine reduces endothelial dysfunction, vasodilate whole vessels and enhance vessel angiogenesis.

There are no previous reports demonstrating effects of sulfasalazine on pro and anti-angiogenic molecules. We show that sulfasalazine not only reduces sFlt- 1 and sENG secretion, but importantly increases PIGF secretion. Sulfasalazine likely reduces sFlt- 1 secretion by decreasing transcription. sFlt- 1 arises from alternative splicing of the Flt- 1 premRNA gene. Two sFlt-1 mRNA variants predominate in preeclampsia; sFlt-1 113 which is widely expressed in many tissues including the endothelium, and the primate specific variant sFlt-1 e15a highly expressed in placenta. Sulfasalazine reduced mRNA expression of sFlt-1 i13 in endothelial cells and e15a in placental explants. Sulfasalazine also reduced sENG secretion, possibly by inhibiting expression of MMP14. MMP14 is thought to cleave membrane bound Endoglin to produce circulating sENG [28]. Our finding of increased PlGF secretion and transcription is exciting because it strengthens the potential of sulfasalazine as a medication for preeclampsia - not only for its capacity to reduce antiangiogenic molecules, but also for its ability to enhance vasoprotective molecules such as PIGF.

We also set out to try to identify the upstream pathways that sulfasalazine may be acting on to reduce sFlt-1 and sENG secretion. We were limited by the fact that the molecular regulation of these factors is still not well understood. Sulfasalazine is known to inhibit $\mathrm{NFKB}$ and also upregulate the antioxidant enzyme HO-1. Despite silencing or overexpressing genes involved in the NFKB pathway in cytotrophoblasts, there was no change in sFlt-1 or sENG secretion suggesting the NFKB pathway may not be involved in their regulation. This is consistent with reports demonstrating the administration of corticosteroids, known to inhibit NFkB [37], do not change serum sFlt-1 and sENG levels in patients with preeclampsia [38]. Furthermore, silencing HO-1 did not affect the ability of sulfasalazine to reduce sFlt-1 secretion from cytotrophoblasts. This is consistent with our previously published findings showing that silencing or upregulating HO-1 in cytotrophoblasts does not affect sFlt-1 or sENG secretion [29]. Therefore, it appears sulfasalazine is not exerting its effects on sFlt-1 and sENG through inhibiting NFKB or upregulating HO-1 pathways and another upstream mechanism, yet to be determined, must be present.

An important feature of a drug that may be useful to treat or prevent preeclampsia would be the ability to reduce endothelial dysfunction.
We have obtained evidence from multiple functional assays using primary cells and tissues to show that sulfasalazine can mitigate endothelial dysfunction. Sulfasalazine is known to reduce markers of inflammation by inhibiting NFKB $[18,39]$. We demonstrated sulfasalazine quenches VCAM-1 expression in HUVECs in the presence of TNF $\alpha$, which is increased in preeclampsia. Furthermore, sulfasalazine enhanced endothelial cell proliferation and migration. Sulfasalazine also induced vasodilation of whole human vessels. It rescued sFlt-1 inhibition of vascular outgrowths from mouse aortic rings. Given these apparent vasoprotective effects of sulfasalazine, further exploration of sulfasalazine as a treatment for cardiovascular conditions may be warranted.

Whether the doses of sulfasalazine used in vitro correspond to the levels achieved in vivo at the level of the placenta is unknown. The in vitro doses of sulfasalazine we used were similar to other studies examining the effect of sulfasalazine on the vasculature [18]. The pharmacokinetics of sulfasalazine in normal healthy individuals would suggest steady state serum concentrations reach around $45 \mu \mathrm{g} / \mathrm{ml}$ [40] when administered at $4 \mathrm{~g}$ per day which corresponds to around $113 \mu \mathrm{M}$ in serum. Therefore we examined the effect of sulfasalazine in vitro at doses straddling this threshold and found sulfasalazine reduced sFlt-1 secretion from cytotrophoblasts. Studies focusing on levels of sulfasalazine in tissues show it concentrates in connective tissues in animal studies $[40,41]$. Unfortunately studies examining its distribution in pregnancy focus on its concentration in the cord blood and breast milk however have not examined the concentrations at the level of the placenta [40]. Therefore we are embarking on a pharmacokinetics study examining the concentration of sulfasalazine at the level of the placenta in patients with preterm preeclampsia (Australia and New Zealand Clinical Trials Registry 12,617,000,226,303).

Sulfasalazine treatment has been linked to renal and liver impairment in case reports. So far there are 5 case reports detailing 6 cases of sulfasalazine induced nephritis. In each case sulfasalazine was administered at $1000 \mathrm{mg}$ daily to $1000 \mathrm{mg}$ three times daily generally for $>12$ months to treat inflammatory bowel disease. In 5 cases the nephritis completely resolved once sulfasalazine was ceased and the patient was commenced on steroids [1-4]. In one case the patient proceeded directly to renal transplant [5]. Reassuringly a review of the side effects of sulfasalazine was performed and despite 4.7 million scripts of sulfasalazine filled in the UK from 1991 to 1998 there were no reports of interstitial nephritis [6]. Furthermore a study examining the effect of long term sulfasalazine use (mean of 10 years) found it to be safe and free of nephrotoxic side effects [7]. Animal studies examining the effect of sulfasalazine on the kidneys revealed that at supraphysiological doses, ten times the dose administered to humans, that interstitial inflammation and oxidative stress occurred [8]. Given the rarity of renal complications coupled with the long-term treatment administered before a complication arose we believe the risk of renal injury is minimal in our population. Given patients with preeclampsia often have renal impairment it is possible that sulfasalazine may in fact improve this if it targets and treats aspects of the preeclampsia disease process. Therefore evaluating renal function will be an important outcome to examine in any trial evaluating the effect of sulfasalazine on preeclampsia treatment or prevention. Sulfasalazine use has also been linked to hepatic dysfunction. In the reported cases all patients experiencing hepatic dysfunction were on concomitant hepatotoxic medications. In the majority of case reports, once sulfasalazine is ceased, the impairment completely resolves. There was one case series involving 2 patients on sulfasalazine that required a liver transplant and one of these patients died a few weeks later [15]. As pregnant patients are not on hepatotoxic medications we believe this complication would be exceedingly rare in preeclamptic patients.

As sulfasalazine mitigates angiogenic factor production and endothelial dysfunction, it holds promise as a potential prevention or treatment for preeclampsia. In contrast to other medications studied that are safe in pregnancy, including proton pump inhibitors [11] and 
metformin [12], sulfasalazine has an added benefit of potentially exerting systemic anti-inflammatory effects. Therefore we believe clinical trials examining its potential as a treatment or prevention may be warranted.

\section{Acknowledgments}

We would like to thank the research midwives, Gabrielle Pell, Genevieve Christophers, Rachel Murdoch and Debra Jinks, and patients at Mercy Hospital for Women for participating in this research.

\section{Declarations of interests}

Dr. Brownfoot, Dr. Hannan, Ms. Cannon, Ms. Nguyen, Dr. Hastie, Dr. Senadheera, Ms. Tuohey, Dr. Tong have nothing to disclose. Dr. Parry reports grants from Ferring Pharmaceuticals, grants from Novartis Pharmaceuticals Australia, outside the submitted work.

\section{Author contributions}

FCB designed and performed experiments, analysed the data and wrote the manuscript. NJH designed experiments and corrected the manuscript. PC, VN and RH performed experiments. LJP, SS designed and performed experiments. TJKL and ST designed experiments, analysed data and assisted with drafting the manuscript.

\section{References}

[1] Redman CW, Sargent IL. Latest advances in understanding preeclampsia. Science 2005;308(5728):1592-4.

[2] Sibai B, Dekker G, Kupferminc M. Pre-eclampsia. Lancet 2005;365(9461):785-99.

[3] Maynard S, Min JY, Merchan J, Lim KH, Li J, Mondal S, et al. Excess placental soluble fms-like tyrosine kinase 1 (sFlt-1) may contribute to endothelial dysfunction, hypertension, and proteinuria in pre-eclampsia. J Clin Invest 2003;111(5):649-58.

[4] Venkatesha S, Toporsian M, Lam C, Hanai J, Mammoto T, Kim YM, et al. Soluble endoglin contributes to the pathogenesis of preeclampsia. Nat Med 2006;12(6): 642-9.

[5] Powe CE, Levine RJ, Karumanchi SA. Preeclampsia, a disease of the maternal endothelium: the role of antiangiogenic factors and implications for later cardiovascular disease. Circulation 2011;123(24):2856-69.

[6] Young BC, Levine RJ, Karumanchi SA. Pathogenesis of preeclampsia. Annu Rev Pathol 2010;5:173-92.

[7] Chaiworapongsa T, Chaemsaithong P, Yeo L, Romero R. Pre-eclampsia part 1: current understanding of its pathophysiology. Nat Rev Nephrol 2014;10(8):466-80.

[8] Cudmore M, Ahmad S, Al-Ani B, Fujisawa T, Coxall H, Chudasama K, et al. Negative regulation of soluble Flt-1 and soluble endoglin release by heme oxygenase-1. Circulation 2007;115(13):1789-97.

[9] Brownfoot FC, Tong S, Hannan NJ, Binder NK, Walker SP, Cannon P, et al. Effects of pravastatin on human placenta, endothelium, and women with severe preeclampsia. Hypertension 2015;66(3):687-97 [discussion 445].

[10] Brownfoot FC, Tong S, Hannan NJ, Hastie R, Cannon P, Kaitu'u-Lino TJ. Effects of simvastatin, rosuvastatin and pravastatin on soluble fms-like tyrosine kinase 1 (sFlt-1) and soluble endoglin (sENG) secretion from human umbilical vein endothelial cells, primary trophoblast cells and placenta. BMC Pregnancy Childbirth 2016;16: 117.

[11] Onda K, Tong S, Beard S, Binder N, Muto M, Senadheera SN, et al. Proton pump inhibitors decrease soluble fms-like tyrosine kinase- 1 and soluble endoglin secretion, decrease hypertension, and rescue endothelial dysfunction. Hypertension 2017 Mar;69 (3):457-68.

[12] Brownfoot FC, Hastie R, Hannan NJ, Cannon P, Tuohey L, Parry LJ, et al. Metformin as a prevention and treatment for preeclampsia: Effects on soluble fms-like tyrosine kinase 1 (sFlt-1) and soluble endoglin secretion, and endothelial dysfunction. Am J Obstet Gynecol March 2016;214(3):356.e1-356.e15.

[13] Cluver CA, Hannan NJ, van Papendorp E, Hiscock R, Beard S, Mol BW, et al. Esomeprazole to treat women with preterm preeclampsia: a randomised placebo controlled trial. Am J Obstet Gynecol 2018 Oct;219(4):388.e1-388.e17.

[14] Benyo DF, Smarason A, Redman CW, Sims C, Conrad KP. Expression of inflammatory cytokines in placentas from women with preeclampsia. J Clin Endocrinol Metab 2001;86(6):2505-12.
[15] Borzychowski AM, Sargent IL, Redman CW. Inflammation and pre-eclampsia. Semin Fetal Neonatal Med 2006;11(5):309-16.

[16] Redman CW, Sacks GP, Sargent IL. Preeclampsia: an excessive maternal inflammatory response to pregnancy. Am J Obstet Gynecol 1999;180(2 Pt 1):499-506.

[17] Sacks GP, Studena K, Sargent K, Redman CW. Normal pregnancy and preeclampsia both produce inflammatory changes in peripheral blood leukocytes akin to those of sepsis. Am J Obstet Gynecol 1998;179(1):80-6.

[18] Kim JY, Cho HJ, Sir JJ, Kim BK, Hur J, Youn SW, et al. Sulfasalazine induces haem oxygenase-1 via ROS-dependent Nrf2 signalling, leading to control of neointimal hyperplasia. Cardiovasc Res 2009;82(3):550-60.

[19] Wahl C, Liptay S, Adler G, Schmid RM. Sulfasalazine: a potent and specific inhibitor of nuclear factor kappa B. J Clin Invest 1998;101(5):1163-74.

[20] Brownfoot FC, Hannan N, Onda K, Tong S, Kaitu'u-Lino T. Soluble endoglin production is upregulated by oxysterols but not quenched by pravastatin in primary placental and endothelial cells. Placenta 2014;35(9):724-31.

[21] Tu'uhevaha J, Kaitu'u-Lino ST, Beard Sally, Hastie Roxanne, Tuohey Laura, Brownfoot Fiona, et al. Characterization of protocols for primary trophoblast purification, optimized for functional investigation of sFlt-1 and soluble endoglin. Pregnancy Hypertens 2014;4(4):287-95.

[22] ACOG. Report of the american college of obstetricians and gynecologists' task force on hypertension in pregnancy. Obstet Gynecol 2013;122:1122-31.

[23] Baker M, Robinson SD, Lechertier T, Barber PR, Tavora B, D'Amico G, et al. Use of the mouse aortic ring assay to study angiogenesis. Nat Protoc 2012;7(1):89-104.

[24] Whitehead CL, Palmer KR, Nilsson U, Gao Y, Saglam B, Lappas M, et al. Placental expression of a novel primate-specific splice variant of sFlt- 1 is upregulated in pregnancies complicated by severe early onset pre-eclampsia. BJOG 2011;118(10): 1268-71.

[25] Kaitu'u-Lino TJ, Tong S, Beard S, Hastie R, Tuohey L, Brownfoot F, et al. Characterization of protocols for primary trophoblast purification, optimized for functional investigation of sFlt-1 and soluble endoglin. Pregnancy Hypertens 2014;4(4):287-95.

[26] Jebbink J, Keijser R, Veenboer G, van der Post J, Ris-Stalpers C, Afink G. Expression of placental FLT1 transcript variants relates to both gestational hypertensive disease and fetal growth. Hypertension 2011;58(1):70-6.

[27] Palmer KR, Tong S, Tuohey L, Cannon P, Ye L, Hannan NJ, et al. Jumonji domain containing protein 6 is decreased in human preeclamptic placentas and regulates sFLT-1 splice variant production. Biol Reprod 2016;94(3):59.

[28] Kaitu'u-Lino TJ, Palmer KR, Whitehead CL, Williams E, Lappas M, Tong S. MMP-14 is expressed in preeclamptic placentas and mediates release of soluble endoglin. Am J Pathol 2012;180(3):888-94

[29] Tong S, Kaitu'u-Lino TJ, Onda K, Beard S, Hastie R, Binder NK, et al. Heme oxygenase1 is not decreased in preeclamptic placenta and does not negatively regulate placental soluble fms-like tyrosine kinase-1 or soluble endoglin secretion. Hypertension 2015:66(5):1073-81.

[30] Austgulen R, Lien E, Vince G, Redman CW. Increased maternal plasma levels of soluble adhesion molecules (ICAM-1, VCAM-1, E-selectin) in preeclampsia. Eur J Obstet Gynecol Reprod Biol 1997;71(1):53-8.

[31] Chaiworapongsa T, Romero R, Yoshimatsu J, Espinoza J, Kim YM, Park K, et al. Soluble adhesion molecule profile in normal pregnancy and pre-eclampsia. J Matern Fetal Neonatal Med 2002;12(1):19-27.

[32] Ahmad S, Ahmed A. Elevated placental soluble vascular endothelial growth factor receptor-1 inhibits angiogenesis in preeclampsia. Circ Res 2004;95(9):884-91.

[33] Mol BW, Roberts CT, Thangaratinam S, Magee LA, de Groot CJ, Hofmeyr GJ. Preeclampsia. Lancet 2016 Mar;387(10022):999-1011.

[34] Skjaerven R, Wilcox AJ, Klungsoyr K, Irgens LM, Vikse BE, Vatten LJ, et al. Cardiovascular mortality after pre-eclampsia in one child mothers: prospective, population based cohort study. BMJ 2012;345:e7677.

[35] Arnadottir GA, Geirsson RT, Arngrimsson R, Jonsdottir LS, Olafsson O. Cardiovascular death in women who had hypertension in pregnancy: a case-control study. BJOG 2005;112(3):286-92.

[36] Irgens HU, Reisaeter L, Irgens LM, Lie RT. Long term mortality of mothers and fathers after pre-eclampsia: population based cohort study. BMJ 2001;323(7323):1213-7.

[37] Barnes PJ. How corticosteroids control inflammation: quintiles prize lecture 2005 . $\mathrm{Br}$ J Pharmacol 2006;148(3):245-54.

[38] Nayeri UA, Buhimschi IA, Laky CA, Cross SN, Duzyj CM, Ramma W, et al Antenatal corticosteroids impact the inflammatory rather than the antiangiogenic profile of women with preeclampsia. Hypertension 2014;63(6):1285-92.

[39] Feeley BT, Park AK, Hoyt EG, Robbins RC. Sulfasalazine inhibits reperfusion injury and prolongs allograft survival in rat cardiac transplants. J Heart Lung Transplant 1999;18(11):1088-95.

[40] Klotz U. Clinical pharmacokinetics of sulphasalazine, its metabolites and other prodrugs of 5-aminosalicylic acid. Clin Pharmacokinet 1985;10(4):285-302.

[41] Hanngren A, Hansson E, Svartz N, Ullberg S. Distribution and metabolism of salicylazo-sulfapyridine. I. A study with C-14-5-amino-salicylic acid. Acta Med Scand 1963; 173:61-72 


\section{University Library}

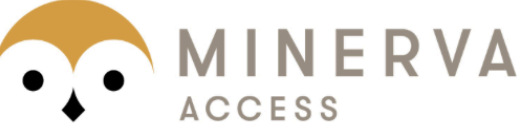

A gateway to Melbourne's research publications

Minerva Access is the Institutional Repository of The University of Melbourne

Author/s:

Brownfoot, FC;Hannan, NJ;Cannon, P;Vi, N;Hastie, R;Parry, LJ;Senadheera, S;Tuohey,

L;Tong, S;Kaitu'u-Lino, TJ

Title:

Sulfasalazine reduces placental secretion of antiangiogenic factors, up-regulates the secretion of placental growth factor and rescues endothelial dysfunction

Date:

2019-03-01

Citation:

Brownfoot, F. C., Hannan, N. J., Cannon, P., Vi, N., Hastie, R., Parry, L. J., Senadheera, S., Tuohey, L., Tong, S. \& Kaitu'u-Lino, T. J. (2019). Sulfasalazine reduces placental secretion of antiangiogenic factors, up-regulates the secretion of placental growth factor and rescues endothelial dysfunction. EBIOMEDICINE, 41, pp.636-648. https://doi.org/10.1016/ j.ebiom.2019.02.013.

Persistent Link:

http://hdl.handle.net/11343/250472

License:

CC BY-NC-ND 